\title{
Estudio epidemiológico de hidatidosis comparando los test de ELISA IgE y Western Blot en población de cinco provincias de la Región Lima. 2015
}

\section{Epidemiological study of hydatidosis comparing IgE ELISA test and Western Blot in population of five provinces of Lima region. 2015}

\author{
Herman Vildozola Gonzales ${ }^{1,2}$, William Roldan Gonzales ${ }^{1,2}$, Yrma Espinoza Blanco ${ }^{2,3}$, Pedro \\ Huapaya Herreros ${ }^{4}$, Susana Jimenez Ramirez ${ }^{2}$, Mercedes Valenzuela Peña ${ }^{5}$, Cristian Villar Huamán ${ }^{5}$ \\ ${ }^{1}$ Universidad Nacional Mayor de San Marcos, Facultad de Medicina, Departamento de Microbiología. Lima, Perú. \\ ${ }^{2}$ Universidad Nacional Mayor de San Marcos, Facultad de Medicina, Instituto de medicina tropical Daniel A. Carrión. Lima, Perú. \\ ${ }^{3}$ Universidad Nacional Mayor de San Marcos, Facultad de Medicina, Departamento de Medicina. Lima, Perú. \\ ${ }^{4}$ Diresa Lima, Ministerio de Salud del Perú. Estrategia Sanitaria Regional de Zoonosis. \\ ${ }^{5}$ Diresa Lima, Ministerio de Salud del Perú. Laboratorio Regional de Referencia en Salud Pública.
}

\section{Correspondencia:}

Herman Vildozola Gonzales

hermanvg62@gmail.com

Universidad Nacional Mayor de San

Marcos. Facultad de Medicina.

Calle Germán Amézaga Nº 375. Lima. Perú.

Recibido: 21 enero 2018

Aceptado: 31 marzo 2018

Conflictos de interés: Ninguno.

Fuentes de financiamiento: Vicerectorado de Investigación y Postgrado de la Universidad Nacional Mayor de San Marcos.

Citar como: Vildozola Gonzales H, Roldan Gonzales W, Espinoza Blanco Y, Huapaya Herreros $P$, Jimenez Ramirez $S$, Valenzuela Peña M, Villar Huamán C. Estudio epidemiológico de hidatidosis comparando los test de ELISA IgE y Westem Blot en población de cinco provincias de la Región Lima. 2015. An Fac med. 2018;79(1):6-10 DOI: http://dx.doi.org/10.15381/anales. v79i1.14585
An Fac med. 2018; 79(1):6-10 / http://dx.doi.org/10.15381/anales.v79i1.14585

\section{Resumen}

Introducción. La aparición de algunas zoonosis en varias provincias de la región Lima en el año 2015, motivó una investigación de diversas zoonosis por la Dirección de Salud de la Región Lima Provincias. Objetivo. Determinar la seroprevalencia de hidatidosis (equinococosis) mediante el test de ELISA IgE comparado con el Western Blot en cinco provincias de la Región Lima. Métodos. Se recolectó un total de 560 muestras de suero de pobladores de cinco provincias de la Región Lima: Canta $(n=180)$, Huaral $(n=55)$, Yauyos $(n=133)$, Cajatambo ( $n=32)$, y Huaura ( $n=160)$. Se detectaron anticuerpos IgE y anticuerpos IgG mediante el test de ELISA y Western-Blot respectivamente. Resultados. Un total de 39 muestras procedentes de pobladores de las provincias de Canta, Yauyos y Huaura, fueron positivas al test de ELISA IgE (seroprevalencia de 6,96\%) y 33 fueron positivas al Western Blot IgG (seroprevalencia de 5,89\%), con presencia de bandas débiles de 8 y $16 \mathrm{kDa}$. Se determinó una concordancia del 99\% entre ambos tests, (indice kappa =0,91). El 50\% (20/39) de los sujetos seropositivos tuvieron entre 25 y 60 años de edad, mientras que la menor proporción de seropositivos (1/39) fueron niños menores de cinco años. Conclusiones: El test de ELISA IgE puede ser utilizado para la detección de individuos seropositivos a anticuerpos anti Echinoccocus spp., mostrando alta concordancia con el Western Blot IgG. Podría utilizarse esta técnica en estudios seroepidemiológicos de campo.

Palabras clave: Zoonosis; Equinococosis; Estudios seroepidemiológicos (fuente: DeCS BIREME).

\section{Abstract}

Introduction. Diagnosis of some zoonosis in different provinces from the Lima region, motivated on epidemiological investigation. Objective. To determine the prevalence of hydatidosis (echinococosis) in population from Lima region by ELISA IgE test compared with Western Blot. Methods. 560 sera samples were collected from people living in five provinces of Lima region: Canta $(n=180)$, Huaral $(n=55)$, Yauyos $(n=133)$, Cajatambo ( $n=32$ ) and Huaura $(n=160)$. Antibodies IgE and IgG were detected by ELISA test and Western Blot respectively. Results. 39 samples were positives to ELISA IgE test (seroprevalence 6,96\%) and 33 samples were positives to Western Blot lgG test (seroprevalence 5,89\%), with light bands of 8 and $16 \mathrm{kDa}$. Positive samples proceeded from people of provinces of Canta, Yauyos and Huaura. Concordancy were determined between both tests (kappa index $=0,91) .50 \%$ (20/39) of positive subjects are from 25 to 60 years old, while less proportion of positives (1/39) were children under 5 years. Conclusions. ELISA IgE test can be used for the detection of positive person to anti Echinococcus spp. antibody, showing very high concordance with Westrn Blot lgG. Could be used in seroepidemiologic studies.

Keywords: Zoonoses; Echinococosis; Seroepidemiologic Studies (source: MeSH NLM). 


\section{INTRODUCCIÓN}

La hidatidosis o equinococosis constituye un serio problema de salud pública en muchas regiones del mundo y es usualmente diagnosticada mediante exámenes clínicos, técnicas de imagen y pruebas inmunológicas. La sensibilidad y especificidad de estas pruebas dependen de la etapa de la enfermedad, la localización de los parásitos, disponibilidad de los antígenos y de las técnicas utilizadas (1-4)

El fluido del quiste hidatídico (HCF por sus siglas en inglés Hydatid cyst fluid) constituye una rica fuente de antígenos para el desarrollo de pruebas inmunológicas para el serodiagnóstico de la hidatidosis. Este fluido contiene al antígeno $5(\mathrm{Ag} 5)$ y el antígeno $\mathrm{B}(\mathrm{AgB})$ que son relevantes para el serodiagnóstico, debido a los altos valores de sensibilidad o especificidad ${ }^{(3,4)}$, así como también para la realización de encuestas epidemiológicas en poblaciones de zonas endémicas o con factores de riesgo. Con el uso de estos antígenos, se ha podido desarrollar el test de ELISA para detección de anticuerpos contra el parásito en pacientes con sospecha clínica de la enfermedad, pudiendo ser confirmada con el test de Western Blot con la aparición de bandas diagnósticas de 8, 16 y 21 kDa ${ }^{(3,4)}$. Una serie de recientes estudios han demostrado el valor potencial que posee la detección de los anticuerpos IgG y el análisis de sus subclases, el dosaje de la IgE total sérica y la detección de anticuerpos IgE en el serodiagnóstico y en el tratamiento ${ }^{(5,6,7)}$. En nuestro medio, se ha estandarizado un test de ELISA para la detección de anticuerpos IgE en pacientes con diferentes formas de hidatidosis, con una alta sensibilidad y especificidad para el serodiagnóstico, además mostrando una buena correlación con la respuesta al tratamiento con albendazol ${ }^{(8)}$.

La región Lima está conformada por nueve provincias del departamento de Lima, a excepción de Lima Metropolitana. En todas las provincias existen zonas rurales donde la actividad principal de la población es la ganadería y, donde paradójicamente, existen escasos registros de las zoonosis asociadas a esta actividad como hidatidosis y que tienen im- portante repercusión económica. En el año 2012, el Ministerio de Salud reportó aproximadamente 1600 casos de hidatidosis que fueron detectados en todo el departamento de Lima, mientras que la Dirección Regional de Salud Lima reportó en el mismo periodo solamente 9 casos de hidatidosis.

Por lo descrito, el objetivo del presente estudio fue determinar la seroprevalencia de anticuerpos anti Echinococcus spp. en la población general de cinco provincias de la región Lima mediante el test de ELISA IgE y comparar sus resultados con el test de Western-Blot.

\section{MÉTODOS}

Se realizó un estudio transversal correlacional. Se consideró como población de estudio a todos los pobladores de cinco provincias de la región Lima mayores de 10 meses, que según estimación del Instituto Nacional de Estadística e Informática para el año 2014 fue de 450,783 habitantes. Considerando un nivel de confianza del $95 \%$ y una precisión del $5 \%$, esperando que el $10 \%$ de los participantes tenga resultado positivo y con la expectativa de un $10 \%$ de pérdidas, el tamaño de muestra necesario fue de 146 personas. Esta muestra fue estratificada por provincias, edades y sexos. Previa información de los objetivos del estudio, todos los participantes dieron su consentimiento informado para ser incluidos en el estudio realizado el año 2015.

Para la preparación de los antígenos utilizados en la serología, se utilizó el fluido hidatídico de Echinococcus granulosus obtenido a partir de quistes hidatídicos hepáticos o pulmonares de origen ovino procedentes de un camal local. El fluido fue centrifugado a 5,000 rpm por cinco minutos, fue dializado contra solución salina y concentrado 100 veces por osmosis inversa. Los antígenos 5 y B fueron semi purificados a partir del fluido hidatídico siguiendo la metodología descrita por Oriol et al. ${ }^{(9)}$. El contenido proteico de los antígenos fue realizado según el método de Lowry ${ }^{(10)}$.

La detección de anticuerpos IgE por el test de ELISA se desarrolló según lo descrito por Vildózola et al. utilizando los antígenos 5 y $\mathrm{B}^{(8)}$. Luego se sensibilizaron placas de poliestireno con los antígenos 5 y $\mathrm{B}$ a una concentración de $1 \mathrm{ug} / \mathrm{mL}$ durante 18 horas a $4^{\circ} \mathrm{C}$ y luego bloqueadas con albúmina sérica bovina al $1 \%$ en buffer fosfato salino por dos horas a $37^{\circ} \mathrm{C}$. Las muestras de suero fueron tratadas con anticuerpos anti IgG humano a una dilución $1 / 20$ y luego centrifugadas para la eliminación de las IgG. Con el objetivo de eliminar los anticuerpos IgG, las muestras de suero fueron incubadas en los pocillos de las placas de poliestireno por 18 horas a $37^{\circ} \mathrm{C}$ y luego fueron lavados tres veces con PBS Tween 20. A continuación, los anticuerpos IgE fueron detectados con anticuerpos anti IgE humano conjugado con peroxidasa diluidos $1 / 1000 \mathrm{e}$ incubados por una hora a $37^{\circ} \mathrm{C}$. Las placas fueron lavadas como ya fue descrito y luego se adicionó una solución de substrato cromógeno (OPD Fast, Sigma Aldrich, USA) y se incubó por 30 minutos a $37^{\circ} \mathrm{C}$. La reacción enzimática fue detenida con la adición de ácido sulfúrico $2.5 \mathrm{~N}$. Las placas fueron leídas en un lector de ELISA a una longitud de onda de 492nm.

Para aplicar el test de Western Blot IgG, los antígenos del fluido hidatídico fueron separados de acuerdo a su peso molecular mediante electroforesis en geles de poliacrilamida en una gradiente de 8 a $20 \%$ y luego fueron electro transferidos a membranas de nitrocelulosa, según el procedimiento descrito por Verástegui et al. ${ }^{(11)}$. Las membranas de nitrocelulosa fueron cortadas en tiras de $3 \mathrm{~mm}$ e inmediatamente utilizadas o almacenadas a $20^{\circ} \mathrm{C}$ hasta el momento de su uso. Para la detección de anticuerpos IgG, cada tira fue incubada con la muestra de suero diluida $1 / 50$ con PBS Tween 20 conteniendo $5 \%$ de leche descremada y fueron incubados por dos horas a temperatura ambiente. Las tiras fueron lavadas tres veces con PBS Tween 20 y luego fueron incubadas con anticuerpos anti IgG humano conjugados con peroxidasa diluidos 1/1000 e incubados por una hora a temperatura ambiente. Las tiras fueron lavadas tres veces con PBS Tween $20 \mathrm{y}$ luego incubadas por tres minutos con una solución cromógeno substrato (DAB en PBS conteniendo peróxido de hidrógeno al 0,01\%). La reacción enzimática fue 
bloqueada con varios lavados con agua destilada. La presencia de por lo menos dos de las tres bandas diagnósticas en la muestra de suero fue considerada confirmatoria de hidatidosis ${ }^{(11)}$.

Para realizar el análisis estadístico, los resultados fueron colectados en una base de datos en el programa Microsoft Excel $^{\circledR} 2013$ (Microsoft Redmond WA) y el cálculo de la sensibilidad, especificidad fue realizado en una tabla de $2 \times 2$ además del valor kappa para evaluar la concordancia entre el test de ELISA IgE y el test de Western Blot IgG. Todos los cálculos fueron realizados con programa SPSS Statistics for windows, versión 15.0 .

\section{RESULTADOS}

Se recolectaron 560 muestras de suero de pobladores de cinco provincias de la región Lima: Canta $(n=180)$, Huaral $(n=55)$, Yauyos $(n=133)$, Cajatambo $(n=32)$, y Huaura $(n=160)$, representando el 23,1\%, 9,82\%, 23,75\%, 5,71\% y $28,57 \%$ de la población estudiada (Tabla 1). En cuanto al género de la población, el $38,4 \%$ correspondió al sexo masculino y el $61,6 \%$ al femenino. En cuanto al género de los individuos seropositivos a anticuerpos IgE, se observó que 15 muestras fueron de pobladores del sexo masculino $(38,5 \%)$ y 24 del femenino $(61,5 \%)$, con una diferencia no significativa (Tabla 2).

La edad promedio de la población estudiada fue 38,97 años, siendo la edad mínima de diez meses y una edad máxima de 95 años. La distribución de la población estudiada según las edades se muestra en la Tabla 3. Un análisis de la seropositividad con respecto a la edad, muestra que el 51,2\% (20/39) de los sujetos seropositivos se encontraban entre los 25 y 60 años, mientras que la menor proporción de seropositivos (1/39) se encuentran entre niños menores de cinco años (Tabla 3).

Un total de 39 de las 560 muestras de suero recolectadas resultaron positivas al test de ELISA IgE, obteniéndose una seroprevalencia del 6,96\% (39/560) para anticuerpos IgE. De estas 39 muestras positivas al test de ELISA IgE, el 84\% (33/39) resultaron ser positivas al test de Wes- tern Blot IgG (presencia de bandas débiles de 8 y 16 kDa), obteniéndose una seroprevalencia de 5,89\% para anticuerpos IgG (33/560) (Tabla 4). Todas estas muestras seropositivas procedían de pobladores de las provincias de Canta, Yauyos y Huaura. El análisis estadístico mostró que existe una concordancia observada del 99\% entre ambas pruebas con un índice kappa de 0,91 (IC 95\% de 0,84-0,98). Sin embargo, el test de ELISA IgE mostró ser más sensible al catalogar a seis individuos como positivos que fueron catalogados como negativos por el test de Western Blot IgG (Tabla 4).

Tabla 1. Serología para hidatidosis en pobladores de cinco provincias de la región Lima mediante el test ELISA IgE y Western Blot IgGpecto de la población con algún problema de salud que no consultó a un establecimiento de salud).

\begin{tabular}{lccccc} 
Provincia & $\begin{array}{c}\mathbf{N}^{\circ} \text { de } \\
\text { muestras }\end{array}$ & $\begin{array}{c}\text { ELISA IgE } \\
\text { Positivo }\end{array}$ & $\%$ & \multicolumn{2}{c}{ Western Blot IgG } \\
Positivo & $\%$ \\
Canta & 180 & 14 & 7,7 & 11 & 6,11 \\
Huaral & 55 & 0 & 0 & 0 & 0 \\
Yauyos & 133 & 15 & 11,28 & 12 & 9,02 \\
Huajatambo & 32 & 0 & 0 & 0 & 0 \\
Total & 160 & 10 & 6,25 & 10 & 6,25 \\
\hline
\end{tabular}

Tabla 2. Serología para hidatidosis según el género de pobladores de cinco provincias de la región Lima mediante el test de ELISA IgE y Western Blot lgG.

\begin{tabular}{lccccc} 
Género & $\mathbf{N}^{\circ}$ de & \multicolumn{2}{c}{ ELISA IgE } & \multicolumn{2}{c}{ Western Blot IgG } \\
& muestras & Positivo & $\%$ & Positivo & $\%$ \\
\hline Masculino & 215 & 15 & 6,98 & 14 & 6,51 \\
Femenino & 345 & 24 & 6,96 & 19 & 5,51 \\
Total & 560 & 39 & 6,96 & 33 & 5,89 \\
\hline
\end{tabular}

Tabla 3. Serología para hidatidosis según la edad mediante el test de ELISA IgE y Western Blot IgG en pobladores de cinco provincias de la región Lima.

\begin{tabular}{lccccc} 
Edad & \multirow{2}{*}{ N $^{*}$ muestras } & $\begin{array}{c}\text { ELISA IgE } \\
\text { Positividad }\end{array}$ & $\%$ & \multicolumn{2}{c}{ Western Blot IgG } \\
& & 1 & 7,14 & 1 & 7,14 \\
\hline a 4 años & 14 & 8 & 8,69 & 8 & 8,69 \\
5 a 14 años & 92 & 5 & 7,14 & 4 & 5,71 \\
15 a 24 años & 70 & 20 & 7,55 & 15 & 5,66 \\
25 a 59 años & 265 & 5 & 4,42 & 5 & 4,42 \\
60 a más & 113 & 0 & 0 & 0 & 0 \\
Sin datos & 6 & 39 & 6,96 & 33 & 5,89 \\
Total & 560 & & &
\end{tabular}

Tabla 4. Concordancia de resultados del test de ELISA IgE respecto a Western Blot IgG en pobladores de cinco provincias de la región Lima $(n=560)$.

\begin{tabular}{lccc} 
ELISA-IgE & \multicolumn{2}{c}{ Western Blot IgG } & Total \\
\hline Positivo & Positivo & Negativo & 39 \\
Negativo & 33 & 6 & 521 \\
Total & 0 & 521 & 560 \\
\hline Indice kappa $=0,91$ (IC 95\% de 0,84-0,98) & 527 &
\end{tabular}


La seroprevalencia general para anticuerpos IgE fue del 6,96\%, mientras que para anticuerpos IgG fue del 5,89\%, que concuerda con lo encontrado por diversos estudios $(16,17,18)$. Con respecto a la seropositividad en cada una de las provincias estudiadas de la Región Lima, se pudo observar que prácticamente todos los sueros positivos para anticuerpos IgE e IgG proceden de las provincias de Canta, Yauyos y Huaura, donde se ha observado que las dos primeras provincias mencionadas se caracterizan por ser zonas rurales y tener a la ganadería como actividad económica predominante ${ }^{(16)}$, lo cual podría explicar los hallazgos al considerarse estas características como potenciales factores de riesgo para la enfermedad ${ }^{(18)}$.

Respecto a la seropositividad de las muestras y su relación con los diferentes rangos de edad, se pudo observar que la mayor proporción de seropositivos se encuentra en individuos de la población económicamente activa, que en el caso de las zonas rurales se caracterizan por mayor contacto con ganado y con perros infectados o de suelo contaminados con huevos del parásito ${ }^{(18)}$.

En relación a las diferencias encontradas en la frecuencia de anticuerpos $\lg E(6,96 \%)$ e IgG $(5,89 \%)$ en el presente estudio, una posible explicación es que se utilizaron antígenos en estado nativo para sensibilizar las placas de ELISA IgE, no han sufrido variación de estructura a diferencia de los antígenos utilizados para la prueba de Western Blot IgG, que son previamente tratados con el detergente SDS a altas temperaturas $\left(95^{\circ} \mathrm{C}\right)$ que desconfigura la estructura tridimensional de las proteínas antigénicas eliminando potencialmente los epítopes conformacionales que normalmente están presentes en el estado nativo del antígeno, quedando expuestos solo los epítopes lineales ${ }^{(19,20)}$.

Una concordancia del 99\% entre la ocurrencia de anticuerpos IgG e IgE demuestra que la mayoría de estos individuos expuestos producen en alguna medida ambos tipos de anticuerpos contra los antígenos del parásito. Sin embargo, hasta el momento se tiene la gran limitación de que en el caso de quistes íntegros o hialinos donde no hay liberación de antígenos
(21) , ya sea de membrana o del fluido hidatídico, no es posible encontrar anticuerpos debido a la pobre o nula estimulación del sistema inmune y a que el diagnóstico confirmatorio finalmente se realiza con la valoración clínica de las imágenes como la ecografía o la resonancia magnética, que a nivel del hígado tiene características muy bien definidas de actividad según la clasificación de la OMS ${ }^{(22)}$

Por otra parte, la sensibilidad de $95,6 \%$ y la especificidad de $100 \%$ para identificar anticuerpos anti IgE de hidatidosis de la prueba de ELISA estandarizada en un estudio anterior ${ }^{(8)}$ se convierte en una alternativa al Western Blot, ya que la positividad es similar, pero la ventaja es que la prueba de ELISA es menos costosa; considerando que en este tipo de enfermedad el precio de las pruebas diagnósticas es elevado y la mayoría de los pacientes que la sufren suelen ser de bajos recursos económicos.

Una limitación del presente estudio fue que no se pudo obtener una cantidad similar de muestras por cada provincia y esto podría ser motivo de sesgo al momento del análisis estadístico.

Se concluye que el test de ELISA IgE puede ser utilizado para la detección de individuos seropositivos a anticuerpos anti Echinococcus spp., mostrando alta concordancia con el Western Blot IgG. Adicionalmente, es posible utilizar esta técnica en estudios de campo para la identificación de sujetos expuestos y probablemente infectados con el parásito. Estos resultados alientan a programar una nueva evaluación para identificar mejor las características clínicas y serológicas, explorando el contenido genético que permita identificar si existen diferencias como el test de Western Blot IgG mostrando una alta concordancia para la detección de individuos seropositivos a anticuerpos anti Echinococcus spp. entre serotipos del parásito en las zonas estudiadas.

\section{REFERENCIAS BIBLIOGRÁFICAS}

1. Eckert J, Deplazes P. Biological, epidemiological, and clinical aspects of echinococcosis, a zoonosis of increasing concern. Clin Microbiol Rev. 2004; 17(1): 107-135. DOI: 10.1128/CMR.17.1.107-135.2004
2. Brunetti $E$, Junghanss T. Update on cystic hydatid disease. Curr Opin Infect Dis. 2009; 22(5):497-502. DOI: $10.1097 /$ CO.0b013e328330331c.

3. Carmena D, Benito A, Eraso E. Antigens for the immunodiagnosis of Echinococcus granulosus infection: An update. Acta Trop. 2006; 98(1):74-86. DOI: https://doi.org/10.1016/j.actatropica.2006.02.002

4. Zhang $\mathrm{W}$, Wen $\mathrm{H}$, Li J, Lin R, McManus DP. Immunology and immunodiagnosis of cystic echinococcosis: an update. Clin Dev Immunol. 2012; 2012:101895. DOI: http://dx.doi. org/10.1155/2012/101895

5. McVie A, Ersfeld K, Rogan MT, Craig PS. Expression and immunological characterization of Echinococcus granulosus recombinant antigen $B$ for lgG4 subclass detection in human cystic echinococcosis. Acta Trop. 1997; 67(12): 1935. DOI: https://doi.org/10.1016/S0001-706X(97)00056-9

6. Daeki AO, Craig PS, Shambesh MK. IgG subclass antibody responses and the natura Ihistory of hepatic cystic echinococcosis in asymptomatic patients. Ann Trop Med Parasitol. 2000; 94(4):319328.

7. Khabiri AR, Bagheri F, Assmar M, Siavashi MR. Analysis of specific $\lg E$ and $\lg G$ subclass antibodies for diagnosis of Echinococcus granulosus. Parasite Immunol. 2006; 28(8): 357-366. DOI: https://doi.org/10.1111/j.1365-3024.2006.00837.x

8. Vildózola H, Espinoza I, Roldan WH. Estandarización de una prueba de ELISA para detectar anticuerpos IgE en pacientes con equinococosis quística y su utilidad en eldiagnóstico y seguimiento de pacientes tratados con albendazol: reporte preliminar. An Fac Med. 2012; 73(1): 35-41. DOI: http://dx.doi.org/10.15381/anales.v73i1.808

9. Oriol R, Williams JF, Pérez Esandi MV, Oriol C. Purification of lipoprotein antigens of Echinococcus granulosus from sheep hydatid fluid. Am J Trop Med Hyg. 1971; 20(4): 569-574.

10. Lowry OH, Rosebrough NJ, Farr AL, Randall RJ. Protein measurement with the Folinphenol reagent. J Biol Chem. 1951; 193(1):265-275.

11. Verastegui M, Moro P, Guevara A, Rodriguez T, Miranda E, Gilman RH. Enzyme linkedimmuno electrotransfer blot test for diagnosis of human hydatid disease. J Clin Microbiol.1992; 30(6):1557-1561.

12. Mihmanli M, Idiz UO, Kaya C, Demir U, Bostanci $\mathrm{O}$, Omeroglu S, et al. Current status of diagnosis and treatment of hepatic echinococcosis. World journal of hepatology. 2016;8(28):1169-81. DOI: 10.4254/wjh.v8.i28.1169

13. García Apaico V. Hernán, Vargas Cuba F, Segovia G, Fernández Chillce I, Miranda E. Seroprevalencia de hidatidosis humana en población adulta de Sancos, Ayacucho 2005. Rev Peru Med Exp Salud Publica. 2009; 26(2): 193-97.

14. Nuñez E, Calero D, Estares L, Morales A. Prevalencia y factores de riesgo de hidatidosis en población general del distrito de Ninacaca Pasco, Perú 2001. An Fac med. 2003; 64(1): 34-42. DOI: http://dx.doi. org/10.15381/anales.v64i1.1419

15. Craig PS, McManus DP, Lightowlers MW, Chabalgoity JA, Garcia HH, Gavidia CM, et al. Prevention and control of cystic echinococcosis. Lancet Infect Dis. 2007; 7(6): 385-94. DOI: 10.1016/S14733099(07)70134-2

16. Chumbe G, Lopera B, Barrón G, Ninaquispe B. Prevalencia de hidatidosis humana mediante técnicas de imagen en Yanahuanca, Pasco. Revista de Investigaciones Veterinarias del Perú. 2010;21(1):61-7. 
17. Gutiérrez WB. Actividades económicas primarias predominantes en la Reserva Paisajística Nor Yauyos Cochas, Perú. Caso: distritos de Huancaya, Alis y Vitis. Apuntes de Ciencia \& Sociedad. 2011;1(2).

18. Possenti A, Manzano-Román R, Sánchez-Ovejero C, Boufana B, La Torre G, Siles-Lucas M, et al. Potential Risk Factors Associated with Human Cystic Echinococcosis: Systematic Review and Meta-analysis. PLOS Neglected Tropical Diseases. 2016;10(11):e0005114. DOI: https://doi. org/10.1371/journal.pntd.0005114
19. Forsström B, Bislawska Axnäs B, Rockberg J, Danielsson H, Bohlin A, Uhlen M. Dissecting Antibodies with Regards to Linear and Conformational Epitopes. PLoS ONE. 2015;10(3):e0121673. DOI: https://doi.org/10.1371/journal.pone.0121673

20. Bass JJ, Wilkinson DJ, Rankin D, Phillips BE, Szewczyk NJ, Smith K, et al. An overview of technical considerations for Western blotting applications to physiological research. Scandinavian journal of medicine \& science in sports. 2017;27(1):4-25. DOI: $10.1111 / \mathrm{sms} .12702$
21. Wuestenberg J, Gruener B, Oeztuerk S, Mason RA, Haenle MM, Graeter T, et al. Diagnostics in cystic echinococcosis: serology versus ultrasonography. Turk J Gastroenterol. 2014;25(4):398-404. DOI: 10.5152/tjg.2014.7112.

22. WHO Informal Working Group. International classification of ultrasound images in cystic echinococcosis for application in clinical and field epidemiological settings. Acta Trop. 2003; 85(2): 253-261. DOI: https://doi.org/10.1016/S0001 706X(02)00223-1 